\title{
Laser-induced photoacoustic tomography for small animals
}

Xueding Wang, Yongjiang Pang, George Stoica, Lihong V. Wang

Xueding Wang, Yongjiang Pang, George Stoica, Lihong V. Wang, "Laserinduced photoacoustic tomography for small animals," Proc. SPIE 4960, Biomedical Optoacoustics IV, (1 July 2003); doi: 10.1117/12.477788

SPIE. Event: Biomedical Optics, 2003, San Jose, CA, United States 


\title{
Laser-induced Photo-acoustic Tomography for Small Animals
}

\author{
Xueding Wang ${ }^{a}$, Yongjiang Pang ${ }^{a}$, George Stoica ${ }^{b}$, and Lihong V. Wang ${ }^{a}$ * \\ a. Optical Imaging Laboratory, Department of Biomedical Engineering, \\ Texas A\&M University, 3120 TAMU, College Station, TX 77843-3120 \\ b. Department of Pathobiology, Texas A\&M University, College Station, TX 77843-5547
}

\begin{abstract}
Photoacoustic tomography, also called opto-acoustic tomography when laser excitation is used, is a novel medical imaging modality that combines the merits of both light and ultrasound. Here, we present our study of laser-induced photoacoustic tomography of organs of small animals. Pulses of $6.5 \mathrm{~ns}$ in width from an Nd:YAG laser at $532 \mathrm{~nm}$ or $1064 \mathrm{~nm}$ are employed to generate the distribution of thermoelastic expansion in the sample. A wide-band ultrasonic transducer that is non-focused in the imaging plane scans around the sample to realize a full-view detection of the imaged cross-section. A modified back-projection algorithm is applied to reconstruct the distribution of optical absorption inside the biological sample. Using optical energy depositions that fall below safe levels, tissue structures in ex-vivo rat kidneys and in-situ mouse brains covered by the skin and skull are imaged successfully with the high intrinsic optical contrast and the high spatial resolution of ultrasound.
\end{abstract}

Keywords: photoacoustic tomography, opto-acoustic tomography, imaging, laser-induced, small animal, backprojection .

\section{INTRODUCTION}

Photoacoustic tomography (PAT) ${ }^{1-7}$, also called thermoacoustic or optoacoustic tomography, is a novel imaging modality that is sensitive not only to different soft tissues with different optical properties but also to functional changes in biological tissues. In PAT, when light energy is absorbed by a biological tissue sample, a small temperature rise in the tissue generates thermoelastic expansion which leads to photoacoustic (PA) waves. The initial thermoelastic pressure is linearly proportional to the local optical absorption (also called optical energy deposition) in the tissue sample. The induced PA waves can be measured using wide-band high-sensitive ultrasonic transducer(s) around the sample. The acquired acoustic signals can then be used to reconstruct the distribution of optical absorption inside the sample. In order to produce PA waves efficiently, the light pulse must be very short. The pulse duration is also a key factor confining the spatial resolution in imaging. This technology, based on intrinsic optical contrast and high ultrasonic resolution, overcomes the resolution disadvantages of pure-optical imaging caused by light diffusion and the contrast and speckle disadvantages of pure-ultrasonic imaging.

\section{EXPERIMENTAL SETUP}

A schematic diagram of our PAT system is shown in Fig. 1, where a laboratory coordinate system [X, Y, Z] is also depicted. An Nd:YAG laser, operating at a wavelength of $532 \mathrm{~nm}$ or $1064 \mathrm{~nm}$ with a FWHM of $6.5 \mathrm{~ns}$ and a pulse repetition rate of $10 \mathrm{~Hz}$, is employed as the light source. The laser beam is expanded and homogenized before it fully illuminates the sample from above along the Z-axis. The distribution of optical absorption in the sample generates proportionate photoacoustic (PA) waves, which are coupled into a high-sensitivity wide-bandwidth ultrasonic transducer that is non-focused in the imaged cross-section in the $X-Y$ plane. The transducer, driven by a computercontrolled step motor to scan around the sample with a $2 \pi$ scanning angle and a step size of $2^{\circ}$, detects the PA signals in the imaging plane at each scanning position. Through a low-noise pulse amplifier and an oscilloscope, the digitized PA signals are collected by a computer for reconstruction and display.

* Author to whom all correspondence should be addressed: phone: 979-847-9040; fax: 979-845-4450; email: LWang@ tamu.edu; http://oilab.tamu.edu. 
In the experiment of imaging the rat kidney ex-vivo, the 1064-nm wavelength is applied. The pulse energy of the laser is controlled to provide an incident energy deposition of about $25 \mathrm{~mJ} / \mathrm{cm}^{2}$ on the kidney surface, which is much lower than the ANSI standards for maximum permissible exposure of pulsed light at the $1064 \mathrm{~nm}$ wavelength. An ultrasonic transducer (V383, Panametrics) that has a bandwidth of $\sim 88 \%$ at $-6 \mathrm{~dB}$ with a center frequency of $3.5 \mathrm{MHz}$ is employed as the PA signal detector. With a cylindrical operating surface and a focal length of $3.2 \mathrm{~cm}$, the transducer is focused along the scanning radius $(4 \mathrm{~cm})$ with a focal diameter of about $1 \mathrm{~mm}$ and a focal zone of about $3 \mathrm{~cm}$, which determines the spatial resolution along the laser axis but not the resolution in the imaging plane.

In the experiment of non-invasive imaging on the mouse brain in situ, the 532-nm wavelength is applied. The incident energy deposition is $<10 \mathrm{~mJ} / \mathrm{cm}^{2}$ on the skin of the mouse head, which induces a temperature rise in the skin estimated to be $<20 \mathrm{mK}$. A non-focused ultrasonic transducer (XMS-310, Panametrics) with a center frequency of 10.4 $\mathrm{MHz}$, a bandwidth of $100 \%$ at $-6 \mathrm{~dB}$, and an active element of $2 \mathrm{~mm}$ in diameter is used as the PA signal detector. The mouse (BALB/c mouse, $40 \mathrm{~g}$, Charles River Breeding Laboratories) is fixed on the jack during the PA signal detection with its head surface in the horizontal plane. Hair remover lotion is used to remove the hair on the mouse head with the skin and skull intact. The transducer scans around the mouse head with a scanning radius of $3.1 \mathrm{~cm}$. The cortical surface of the mouse is placed in the scanned cross-section.

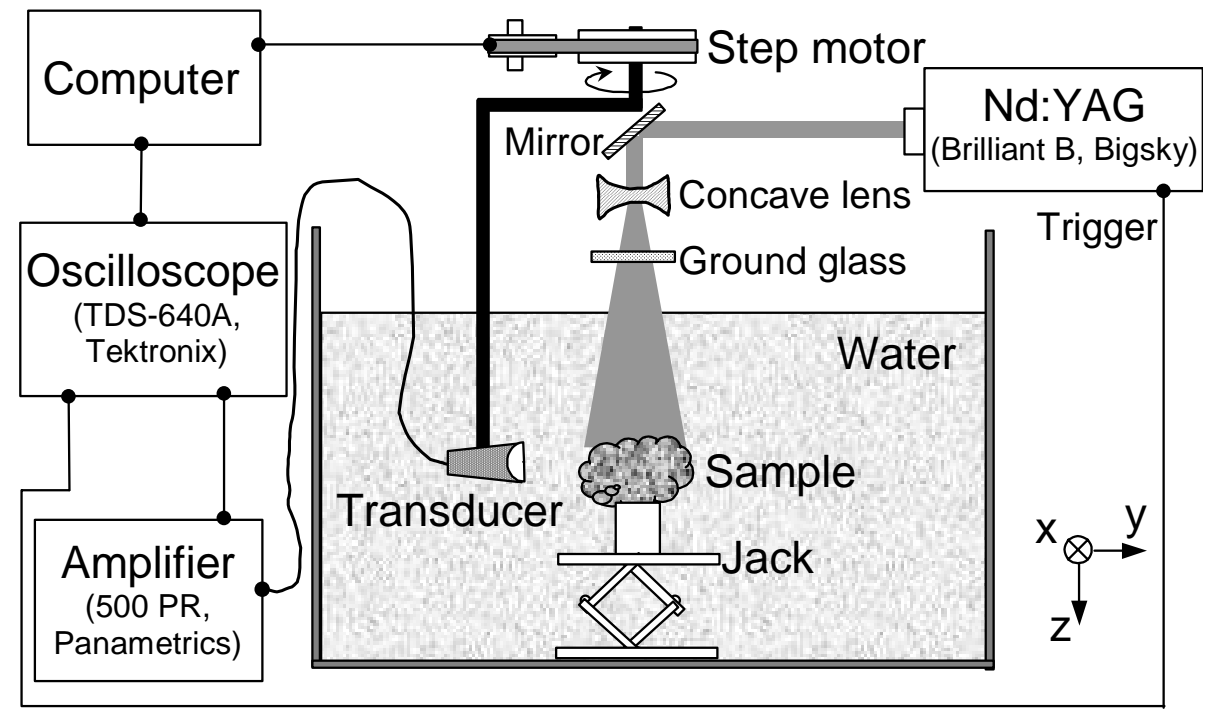

Fig. 1. The experimental setup for photoacoustic tomography.

\section{RECONSTRUCTION ALGORITHM}

For the PA effect in our system, heat conduction can be neglected because the duration of the laser pulse is much shorter than the thermal diffusion time ${ }^{8}$. Our recent development of an accurate reconstruction algorithm ${ }^{9}$ as well as the configuration of laser illumination relative to the acoustic detection plane in combination with the full-view ( $2 \pi$ ) detection of the imaged cross-section plays a crucial role in the effort reported here. For cases where the scanning radius in a circular-scan configuration is much greater than the PA wavelengths (true in our experiments), we have

$$
A(\mathbf{r})=-\left.\frac{r_{0}^{2} C_{p}}{2 \pi v_{s}^{4} \beta} \int_{\theta_{0}} d \theta_{0} \frac{1}{t} \frac{\partial p\left(\mathbf{r}_{0}, t\right)}{\partial t}\right|_{t=\left|\mathbf{r}_{0}-\mathbf{r}\right| / v_{s}},
$$

where $A(\mathbf{r})$ denotes the optical absorption within the sample at position $\mathrm{r} ; C_{p}$ is the specific heat; $v_{s}$ is the acoustic speed; $\beta$ is the thermal coefficient of volume expansion; $\mathbf{r}_{0}$ is the detector position with respect to the imaging center; and $p\left(\mathbf{r}_{0}, t\right)$ is the PA signals detected at each scanning angle $\theta_{0}$. 


\section{IMAGING RESULTS}

\subsection{Imaging of the rat kidney $e x$-vivo}

We apply our PAT system to imaging rat kidneys ex-vivo, because kidney is a good biological organ model with several tissue layers and distinctive interior structures. Fig. 2a shows the PAT image of the center layer of a rat kidney where the optical absorption is depicted in gray scale (darker areas represent tissues with higher absorption). The size of the rat kidney is about $1.2 \mathrm{~cm} \times 0.8 \mathrm{~cm}$ in the imaging plane, and the thickness of the kidney along the Z-axis is $\sim 0.6 \mathrm{~cm}$. The imaged cross-section is $\sim 0.3 \mathrm{~cm}$ below the upper surface of the kidney. By comparing the reconstructed image and the anatomy picture (Fig. 2b), we see that the features of the interior tissues, including the renal pelvis and the calyces, are classified with satisfactory contrast and resolution. Fig. 2c shows the PAT image of another rat kidney of similar size where the imaged cross-section is $\sim 1 \mathrm{~mm}$ below the upper surface of the kidney. The reconstructed absorption distribution matches well with the interior tissue structures shown by the corresponding anatomy picture (Fig. 2d). Three layers of soft tissues, which are recognized to be the cortex, the cuter medulla and the inner medulla, are imaged with good contrast.

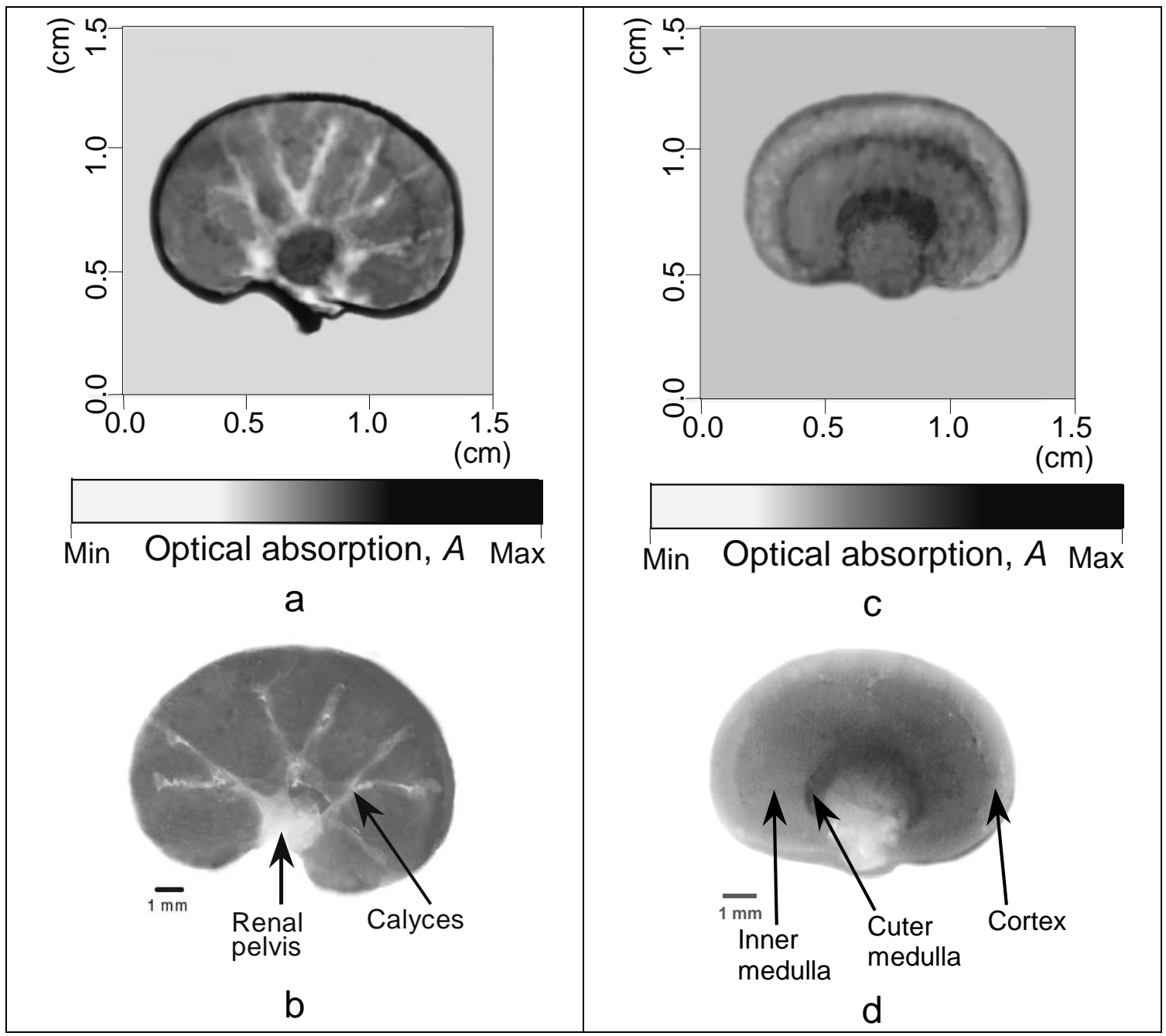

Fig. 2. (a) The photoacoustic image and (b) the corresponding anatomy picture of the interior tissue structure of a rat kidney, where the imaged cross-section is the center layer of the kidney. (c) The photoacoustic image and (d) the corresponding anatomy picture of the interior tissue structure of another rat kidney, where the imaged cross-section is about $1 \mathrm{~mm}$ below the upper surface of the kidney. 


\subsection{Non-invasive imaging of the mouse brain}

PAT imaging of the mouse brain has been achieved non-invasively with the skin and skull intact, as shown in Fig. 3. The optical absorption is depicted in gray scale, where the darker areas — such as the blood vessels - have comparatively higher absorption. Due to different optical absorption, the various soft tissues are identified clearly. The detailed brain structures are indicated in Fig. 3b. The smallest vessels in the superficial cortex that can be seen by this PAT system have a diameter of $\sim 30 \mu \mathrm{m}$, which presents a width of $\sim 90 \mu \mathrm{m}$ in the images. This shows that the spatial resolution of this PAT system with a $10 \mathrm{MHz}$ transducer is $\sim 60 \mu \mathrm{m}$. This resolution approaches the diffraction limit of the detected PA waves. ${ }^{10}$ This also demonstrates that the resolution of PAT is limited by the bandwidth of the detected PA signals rather than by optical diffusion as is the case in pure-optical imaging.

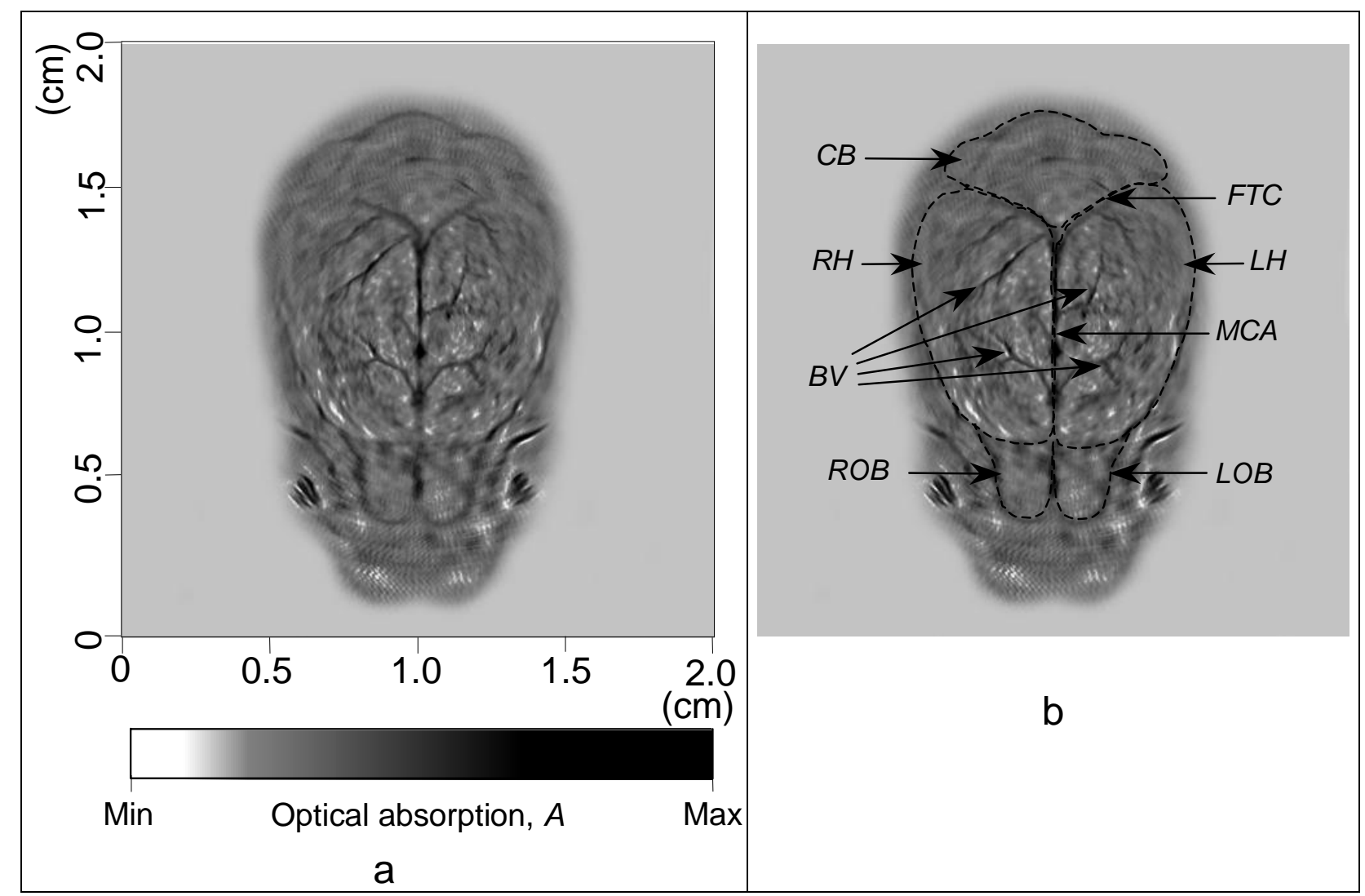

Fig. 3. (a) The photoacoustic image of the superficial layer of a mouse brain, which is achieved non-invasively with the skin and skull intact. The size of the brain is about $1.0 \mathrm{~cm} \times 1.5 \mathrm{~cm}$ in the imaged cross-section. The detailed brain structures can be visualized clearly and accurately. Detailed organizations that can be classified are indicated in (b): $B V$ : blood vessel; $C B$ : cerebellum; $F T C$ : fissura transversa cerebri; $L H$ : left hemisphere; $L O B$; left olfactory lobe; $M C A$ : middle cerebral artery; $R H$ : right hemisphere; and $R O B$ : right olfactory lobe.

\section{CONCLUSIONS}

In summary, this study demonstrates applications of our PAT system based on full-view detection of an imaged crosssection and a high-accuracy modified back-projection algorithm. Various tissues within the rat kidney are visualized ex-vivo using safe levels of optical energy deposition. Detailed tissue structures in the mouse brain are also mapped non-invasively with the skin and skull intact. The PAT technique presented in this paper offers promise for imaging and classifying various soft tissues in biological organs with intrinsic optical contrast and high spatial resolution. 


\section{ACKNOWLEDGMENTS}

We thank G. Ku for experimental assistance. The research was supported in part by DOD, NIH, NSF, and Texas ARP.

\section{REFERENCES}

1. C. G. A. Hoelen, F. F. M. de Mul, R. Pongers and A. Dekker, "Three-dimensional photoacoustic imaging of blood vessels in tissue," Opt. Lett. 23, pp.648-650, 1998.

2. R. A. Kruger, D. R. Reinecke and G. A. Kruger, "Thermoacoustic computed tomography-technical considerations," Med. Phys. 26, pp.1832-1837, 1999.

3. R. O. Esenaliev, A. A. Karabutov and A. A. Oraevsky, "Sensitivity of laser opto-acoustic imaging in detection of small deeply embedded tumors," IEEE J. Sel. Top. Quant. 5, pp.981-988, 1999.

4. G. Paltauf and H. Schmidt-Kloiber, "Optical method for two-dimensional ultrasonic detection," Appl. Phys. Lett. 75, pp.1048-1050, 1999.

5. A. A. Karabutov, E. V. Savateeva and N. B. Podymova, "Backward mode detection of laser-induced wide-band ultrasonic transients with optoacoustic transducer," J. Appl. Phys. 87, pp.2003-2014, 2000.

6. K. P. Köstli, D. Frauchiger, J. J. Niederhauser, G. Paltauf, H. P. Weber and M. Frenz, "Optoacoustic imaging using a three-dimensional reconstruction algorithm," IEEE J. Sel. Top. Quant. 7, pp.918-923, 2001.

7. X. Wang, Y. Pang, G. Ku, X. Xie, G. Stoica and L. V. Wang, "Non-invasive laser-induced photoacoustic tomography of the brain in vivo: structural and functional neuroimaging," Nat. Biotech., accepted.

8. G. J. Diebold, T. Sun and M. I. Khan, "Photoacoustic and photothermal phenomena III," (D. Bicanic, eds.), pp. 263-296, Springer-Verlag, Berlin, Heidelberg, 1992.

9. M. Xu and L. V. Wang, "Time-domain reconstruction for thermoacoustic tomography in a spherical geometry," IEEE T. Med. Imaging 21, pp.814-822, 2002.

10. M. Xu and L. V. Wang, "An analytic explanation of spatial resolution related to bandwidth and detector aperture size in thermoacoustic or photoacoustic reconstruction," Phys. Rev. E, accepted. 